\title{
EFFECT OF VENIPUNCTURE SITE ON HEMATOLOGIC AND SERUM BIOCHEMICAL PARAMETERS IN MARGINATED TORTOISE (TESTUDO MARGINATA)
}

\author{
Jorge Ramón López-Olvera, ${ }^{1}$ Jordi Montané, ${ }^{1}$ Ignasi Marco, ${ }^{1,3}$ Albert Martínez-Silvestre,${ }^{2}$ \\ Joaquim Soler, ${ }^{2}$ and Santiago Lavín ${ }^{1}$ \\ 'Servei d'Ecopatologia de Fauna Salvatge, Facultat de Veterinària, Universitat Autònoma de Barcelona, 08193- \\ Bellaterra, Barcelona, Spain \\ ${ }^{2}$ Centre de Recuperació d'Amfibis i Rèptils de Catalunya (C.R.A.R.C.), 08783-Masquefa, Barcelona, Spain \\ ${ }^{3}$ Corresponding author (email: Ignasi.Marco@uab.es)
}

ABSTRACT: Blood samples were obtained from the dorsal coccygeal vein and the brachial vein of five adult (four females and one male) and two subadult males of marginated tortoise (Testudo marginata) and hematologic and biochemical parameters were compared. Significant differences were found for red blood cell count, hematocrit, hemoglobin concentration, total proteins, uric acid, aspartate aminotransferase, alanine aminotransferase, lactate dehydrogenase, alkaline phosphatase, calcium, and phosphorus, which were greater in the brachial vein samples. Hemodilution due to lymph was observed when collecting blood from the dorsal coccygeal vein, and it is thought to be the cause of the differences found. This research documented that the brachial vein is a more reliable and consistent venipuncture site than dorsal coccygeal vein in marginated tortoise.

Key words: Chelonians, hematology, marginated tortoise, serum chemistry, Testudo marginata.

\section{INTRODUCTION}

Hematologic and blood biochemical parameters are essential to assess health and clinical status of reptiles, and particularly in tortoises, due to the fact that these animals demonstrate few observable clinical signs and the diagnostic challenges of evaluating them (Gottdenker and Jacobson, 1995). Establishment of hematologic and blood biochemical reference values and standardization of sample collection methods in reptiles may be useful in many fields, ranging from exotic animal practice to wildlife rehabilitation centers (Muro et al., 1994; Raphael et al., 1994). Also, monitoring reptile blood parameters can be a way to manage and evaluate the physiologic and health status of their populations, and may be a useful indicator of the environmental status, because these species are very sensitive to habitat changes (Jacobson et al., 1991; Raphael et al., 1994; Dickinson et al., 2002).

However, many factors (both intrinsic and extrinsic) make it difficult to establish reference blood values for a species. Some of the most important intrinsic factors are species, sex, age, and physiologic status.
Among the extrinsic factors are season, temperature, habitat, diet, and captivity (Lawrence and Hawkey, 1986; Gottdenker and Jacobson, 1995). The site and method of blood sample collection are also extrinsic factors that may influence hematologic and serum biochemical values. Standardization of blood collection techniques and sites will improve accuracy of blood data measurement and comparison (Gottdenker and Jacobson, 1995).

Blood collection sites and methods in tortoises described included the jugular vein, dorsal coccygeal vein, brachial vein, lateral coccygeal vein, postoccipital venous plexus, femoral venous plexus, orbital sinus, heart, and nail-clipping (Samour et al., 1984; Marks and Citino, 1990; Gottdenker and Jacobson, 1995; Murray, 2000). The jugular vein, especially the right one, has been reported as the preferred site for venipuncture in some tortoise species (Gottdenker and Jacobson, 1995; Jenkins, 1996; Lloyd and Morris, 1999), but according to other authors the dorsal coccygeal vein and the brachial vein are preferred, particularly in Testudo (Samour et al., 1984; Marks and Citino, 1990; 
Jackson, 1991; Göbel and Spörle, 1991; Muro et al., 1994; Murray, 2000). However, risk of contamination of blood samples with lymph due to proximity of lymphatic and blood vessels has been described for the brachial vein (Jacobson et al., 1992; Gottdenker and Jacobson, 1995; Murray, 2000) and the dorsal coccygeal vein (Marks and Citino, 1990; Jacobson et al., 1992; Muro et al., 1994).

The objective of this study was to analyze the effect of sample collection site from the dorsal coccygeal vein and the brachial vein on hematologic and serum biochemical parameters in the marginated tortoise (Testudo marginata).

\section{MATERIALS AND METHODS}

Paired blood samples were collected from the dorsal coccygeal vein and the brachial vein of five adult (four females and one male) and two subadult male marginated tortoises. Animals were physically restrained to permit blood collection. Body weight ranged from 1,085 to 4,844 g, and shell length measured along the midline ranged from 199 to $326 \mathrm{~mm}$. Tortoises were kept in captivity in open air enclosures at the Catalonian Reptile and Amphibians Rehabilitation Center (CRARC) in Masquefa (Barcelona, Spain, $41^{\circ} 30^{\prime} \mathrm{N} 1^{\circ} 48^{\prime} \mathrm{E}$ ), receiving $14 \mathrm{hr}$ of light and $10 \mathrm{hr}$ of darkness a day. Tortoises were fed a diet of fruit and vegetables and had access to spontaneous autochthonous plants. All blood samples were obtained between May and June of 2001, to avoid the effect of season and physiologic status on blood parameters. Mean temperature was $20.6 \mathrm{C}$, with a daily oscillation of $13.6 \mathrm{C}$ and mean humidity oscillated between $52 \%$ and $59 \%$.

Blood samples were collected in $2 \mathrm{ml}$ disposable plastic syringes with 23 gauge needles. One milliliter of blood was placed immediately in lithium heparin tubes for hematologic analysis, whereas the remaining was placed in another tube without anticoagulant and allowed to clot at room temperature, then centrifuged at $1,200 \times \mathrm{G}$ for $10 \mathrm{~min}$ and serum frozen at $-20 \mathrm{C}$ until used for biochemical determinations.

Hematologic analysis included red blood cell count (RBC), hematocrit (PCV), hemoglobin concentration, mean corpuscular volume (MCV), mean corpuscular hemoglobin $(\mathrm{MCH})$, mean corpuscular hemoglobin concentration (MCHC), white blood cell count (WBC) and leukocyte differential count. Hematocrit was determined with a microhematocrit centrifuge (Hawksley, Lancing, $\mathrm{UK}$ ) at $14,000 \times \mathrm{G}$ for $5 \mathrm{~min}$; hemoglobin concentration was determined by a semiautomatic hematologic analyzer (Sysmex F-800, Toa Medical Electronics Co. Ltd, Japan) after centrifugation of lysates. Blood cell counts were determined in a Neubauer hemocytometer chamber with Natt \& Herrick's solution as described (Raskin, 2000). Differential leukocyte count was performed on a total of $100 \mathrm{WBC}$ on blood smears with a Diff-Quick-like stain (Química Clínica Aplicada S.A., Amposta, Spain).

Biochemical parameters included total protein, glucose, triglycerides, urea, creatinine, uric acid, calcium, phosphorus, creatine kinase (CK), aspartate aminotransferase (AST), alanine aminotransferase (ALT), lactate dehydrogenase (LDH), and alkaline phosphatase (ALP), and were determined by means of an automated analyzer (COBAS MIRA, Roche, Nutley, New Jersey, USA). Sodium was determined with a flame spectrophotometer (Corning 410C, Corning Medical, Medfield, Massachusetts, USA).

Complete hematologic data from all the animals were available, whereas biochemical data for both venipuncture sites were available from only three to six animals, due to the lack of enough serum. Mean, standard deviation, coefficient of variation, and bias percentage were determined for each venipuncture site. A Shapiro-Wilk test was used to assess normality of the data. Hematologic and biochemical parameters were compared using a paired Student's $t$-test and a Wilcoxon's test, depending on the distribution of the values for each parameter. Statistical and distribution analyses were performed using a software program (SAS, SAS Institute Inc., Cary, USA).

\section{RESULTS}

Significant differences $(P \leq 0.05)$ were found between dorsal coccygeal vein and brachial vein values for RBC, PCV, and hemoglobin concentration, which were higher in the samples collected from the brachial vein. Coefficient of variation was greater in dorsal coccygeal vein samples for these three parameters. The relative bias of dorsal coccygeal vein samples was at least $30 \%$ for all the hematologic parameters, except percentage leukocyte differential count and $\mathrm{MCV}, \mathrm{MCH}$, and $\mathrm{MCHC}$ (Table 1).

Total protein, uric acid, calcium, phosphorus, AST, ALT, LDH, and ALP were 


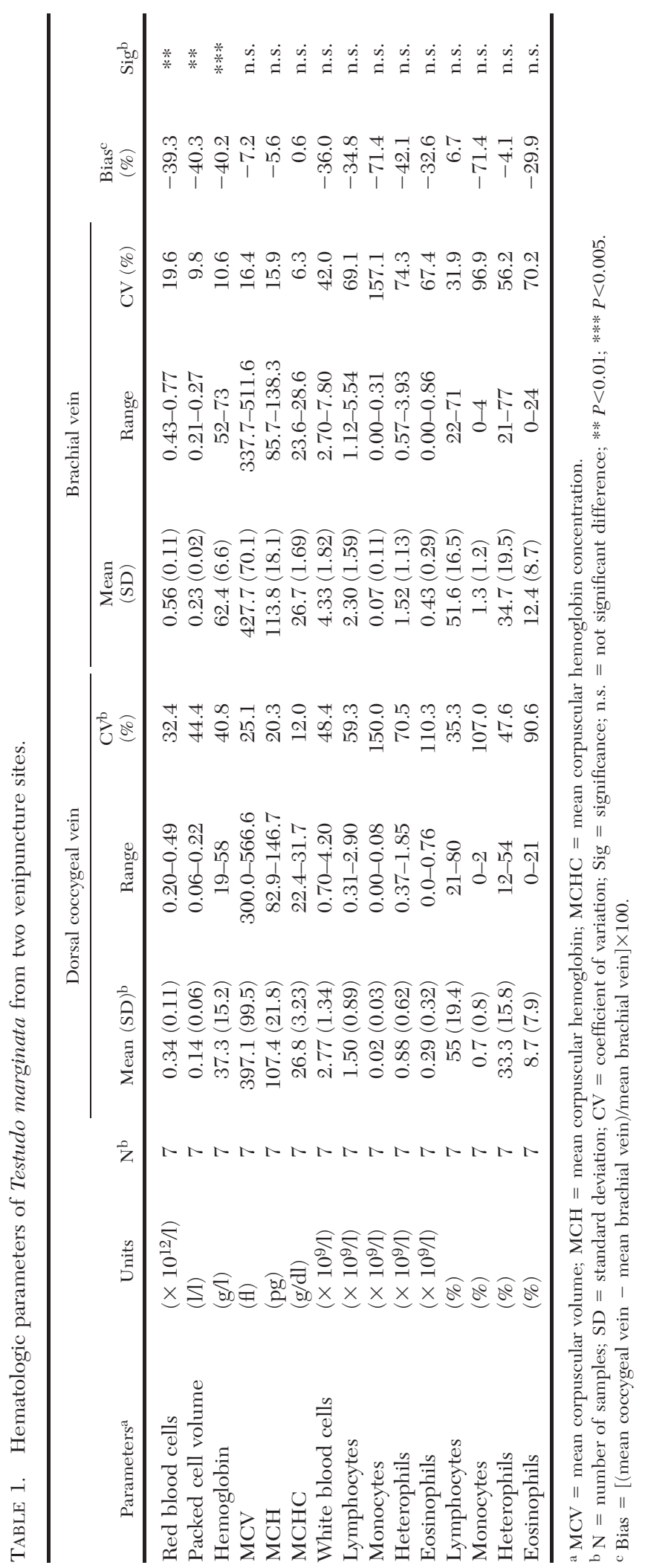


greater in the brachial vein samples than in the dorsal coccygeal vein samples $(P \leq 0.05)$. Coefficient of variation was also greater in dorsal coccygeal vein samples for all the biochemical parameters where significant differences were found, except calcium. Urea, creatinine, and CK, which were not significantly different between both venipuncture sites, showed also a greater variability (coefficient of variation) in dorsal coccygeal vein samples. The relative bias of dorsal coccygeal vein samples was at least $20 \%$ for all the biochemical parameters, except sodium (Table 2).

\section{DISCUSSION}

Blood samples from different venipuncture sites have been used to establish reference values in different species of tortoises, without finding any significant difference in the parameters studied: dorsal coccygeal vein and nail-clipping (Lawrence and Hawkey, 1986); jugular vein and brachial vein (Marks and Citino, 1990); and dorsal coccygeal vein and brachial vein (Göbel and Spörle, 1992). Venipuncture or blood collection site is important to consider when interpreting blood analysis results to assess health status and to establish reference values, because blood values can vary considerably (Gottdenker and Jacobson, 1995). In chelonians and other ectotherms, it is particularly difficult to establish reference intervals, because they are capable of profound physiologic adaptation to different habitats, seasons, and environmental changes. Different reference intervals must be considered depending on age, sex, geographic location, season, and other environmental factors (Christopher et al., 1999). Minimum information about the reference population must be provided when establishing a reference interval, in order to ensure that physiologic data are obtained in the same circumstances. Even then, up to 97 samples from a population are required to develop statistically meaningful intervals (Walton, 2001). Reference values for hematologic and biochemical parameters have been previously established for marginated tortoise from brachial vein blood samples (Martínez-Silvestre et al., 2001).

Differences in the values of blood samples collected from different venipuncture sites have been described in desert tortoises (Gopherus agassizii). Lymph contamination of the blood samples collected from the postoccipital venous plexus, in contrast to samples obtained from the jugular vein, was found to be a causal factor (Gottdenker and Jacobson, 1995). Blood sampling in a vessel other than the jugular vein or the carotid artery (the only peripheral vessels that can be visualized through the skin in medium-sized tortoises) is always blind and may result in lymph contamination (Jacobson et al., 1992; Lloyd and Morris, 1999). Such contamination has been described when collecting blood samples from the brachial vein in desert tortoises (Gottdenker and Jacobson, 1995). Nevertheless, the brachial vein has been preferred to jugular venipuncture to collect blood samples in the radiated tortoise (Geochelone radiata), since the axillary approach was more consistent and less invasive and stressful (Marks and Citino, 1990). Collecting blood samples from the jugular vein or the carotid artery requires a cooperating animal or manual extension and restraint of the head, and may be difficult or even impossible in some individuals (Jacobson et al., 1992; Lloyd and Morris, 1999). Overall, it is easier to sample from the brachial vein than from the carotid artery or the jugular vein.

Differences in values obtained in our study between the brachial vein and dorsal coccygeal vein can also be explained by lymph contamination of the samples collected from the dorsal coccygeal vein. Lymph contamination of the samples was clearly seen in five of the seven animals when collecting blood from this vessel. We observed a transparent fluid coming first into the syringe, mixing after a few seconds with blood inside the syringe. However, we did not observe this macroscopic contamination in any of the samples ob- 


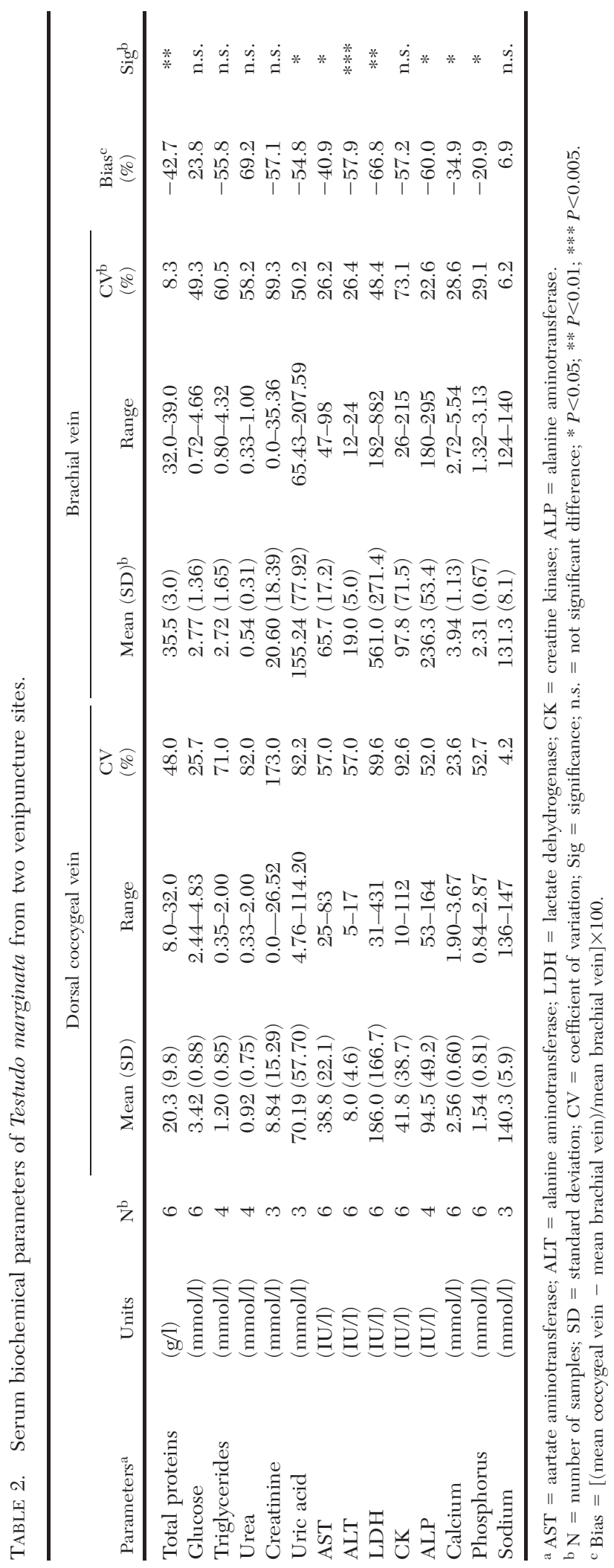


tained from the brachial vein. Although minor contamination of brachial vein samples could have happened, the normal and narrow distribution of blood values of these samples indicates lack of lymph contamination.

Lymph composition is different from blood and serum, so contamination of blood samples with lymph may affect the values of hematologic and biochemical parameters. Volume percentages of cellular components are lower in lymph than in blood, and enzyme activities and concentration of total protein are lower in lymph than in serum, whereas chloride values are higher (Jacobson et al., 1992; Gottdenker and Jacobson, 1995).

Differences observed between samples collected from the dorsal coccygeal vein and from the brachial vein for RBC, PCV, hemoglobin concentration, total protein, uric acid, AST, ALP, calcium, and phosphorus, can be explained as a results of lymph contamination similar to that described in desert tortoises (Gottdenker and Jacobson, 1995). We also found differences in $\mathrm{LDH}$, which was not analyzed in the aforementioned study. In contrast with their results, we detected significantly different values for ALT, but not for leukocytes (WBC, heterophils, and monocytes) or glucose. The high interindividual variability in total and differential leukocyte counts could explain the absence of significant difference in these parameters.

The values of brachial vein samples were not only greater, but also less variable for most of the hematologic and serum biochemical parameters analyzed. This probably reflects the effect of lymph hemodilution in dorsal coccygeal samples, since lymph contamination of these blood samples varied considerably. The elevated bias of dorsal coccygeal vein samples for most hematologic and biochemical parameters also may be due to lymph hemodilution. The only parameters that showed less bias from this vessel were those which are not affected by lymph hemodilution:
MCV, MCH, MCHC, percentage leukocyte differential count, and sodium.

The results obtained in our study indicated that venipuncture site has an important influence on hematologic and biochemical values in the marginated tortoise. According to our results, the brachial vein is a more reliable and consistent venipuncture site than the dorsal coccygeal vein in marginated tortoise, although dorsal coccygeal vein has been considered the site of choice for venipuncture in tortoises of the Genus Testudo (Jackson, 1991; Muro et al., 1994; Murray, 2000). Interpretation of hematologic and serum biochemical analysis requires the existence of reference values and the standardization of sample collection and analysis techniques (including venipuncture site), so results of different origin can be compared. Further studies comparing hematologic and biochemical values of marginated tortoise at different collection sites (jugular vein, ventral coccygeal vein, postoccipital venous plexus, subcaparacial vein, femoral area, lateral coccygeal vein) would serve to determine suitability and effects on the hematologic and biochemical parameters of each venipuncture site, to establish separate reference intervals, and to standardize blood sample collection and handling techniques.

\section{LITERATURE CITED}

Christopher, M. M., K. H. Berry, I. R. Wallis, K. A. Nagy, B. T. Henen, and C. C. Peterson. 1999. Reference intervals and physiologic alterations in hematologic and biochemical values in the Mojave desert. Journal of Wildlife Diseases 35: 212-238.

Dickinson, V. M., J. L. JARChOW, AND M. H. TruebloOd. 2002. Hematology and plasma biochemistry reference range values for freeranging desert tortoises in Arizona. Journal of Wildlife Diseases 38: 143-153.

GÖBEL, T., AND H. SPÖRLE. 1991. Blood collecting technique and selected reference values for Herman's tortoise (Testudo hermanni hermanni). In Proceedings: 4th International colloquium on pathology and medicine of reptiles and amphibians, German Veterinary Association, Bad Nauheim, Germany, pp. 129-134.

, AND 1 1992. Blutentnahmetechnik 
und Serumnormalwerte wichtiger Parameter bei der griechischen Landschildkrote (Testudo hermanni hermanni). Tierarztliche Praxis 20: 231234.

Gottdenker, N. L., AND E. R. JaCobson. 1995. Effect of venipuncture sites on hematologic and clinical biochemical values in desert tortoises (Gopherus agassizii). American Journal of Veterinary Research 56: 19-21.

JACKSON, O. F. 1991. Chelonians. In Manual of exotic pets, P. H. Beynon and J. E. Cooper (eds.). British Small Animal Veterinary Association, Cheltenham, UK, pp. 221-243.

Jacobson, E. R., J. M. Gaskin, M. B. Brown, H. K. Harris, C. H. Gardiner, J. L. Lapointe, H. P. Adams, and C. Reggiardo. 1991. Chronic upper respiratory tract disease of free-ranging desert tortoises (Xerobates agasizii). Journal of Wildlife Diseases 27: 296-316.

, J. Schumacher, and M. Green. 1992. Field and clinical techniques for sampling and handling blood for hematologic and selected biochemical determinations in desert tortoise, $\mathrm{X} e$ robates agassizii. Copeia 1: 237-241.

Jenkins, J. R. 1996. Diagnostic and clinical techniques. In Reptile medicine and surgery, D. R. Mader (ed.). W. B. Saunders Company, Philadelphia, pp. 264-276.

LaWrence, K., AND C. HaWkey. 1986. Seasonal variations in haematological data from Mediterranean tortoises (Testudo graeca and Testudo hermanni) in captivity. Research in Veterinary Science 40: 225-230.

LLOYD, M., AND P. Morris. 1999. Chelonian venipuncture techniques. Bulletin of the Association of Reptilian and Amphibian Veterinarians 9: 2629.
Marks, S. K., AND S. B. Citino. 1990. Hematology and serum chemistry of the radiated tortoise (Testudo radiata). Journal of Zoo and Wildlife Medicine 21: 342-344.

Martínez-Silvestre, A., S. Lavín, I. Marco, J. Montané, J. R. López, and J. Soler-Massana. 2001. Haematology and plasma chemistry of captive Testudo marginata. In Proceedings: International congress on Testudo Genus, B. Devaux (ed.). Editions SOPTOM, Hyères, France, pp. 187-189.

Muro, J., R. CuenCA, L. ViÑas, AND S. LAVín. 1994. Interés del hemograma en la clínica de quelonios. Veterinaria en Praxis 9: 24-29.

Murray, M. J. 2000. Reptilian blood sampling and artifact considerations. In Laboratory medicine: Avian and exotic pets, A. M. Fudge (ed.). W. B. Saunders Company, Philadelphia, pp. 185-192.

Raphael, B. L., M. W. Klemens, P. Moehlman, E. DIERENFELD, AND W. B. KaRESH. 1994. Blood values in free-ranging pancake tortoises (Malacochersus tornieri). Journal of Zoo and Wildlife Medicine 25: 63-67.

RASKIN, R. E. 2000. Reptilian complete blood count. In Laboratory medicine: Avian and exotic pets, A. M. Fudge (ed.). W. B. Saunders Company, Philadelphia, pp. 193-197.

Samour, H. J., D. Risley, T. March, B. Savage, O. NiEva, AND D. M. Jones. 1984. Blood sampling techniques in reptiles. The Veterinary Record 114: 472-476.

WALtON, R. M. 2001. Establishing reference intervals: Health as a relative concept. Seminars in Avian and Exotic Pet Medicine 10: 66-71.

Received for publication 30 December 2002. 